Proceedings of the 40th Annual Meeting of the Association for Computational Linguistics (ACL), Philadelphia, July 2002, pp. 183-190.

\title{
Exploring Asymmetric Clustering for Statistical Language Modeling
}

\author{
Jianfeng Gao \\ Microsoft Research, Asia \\ Beijing, 100080, P.R.C \\ jfgao@microsoft.com \\ Guihong $\mathrm{Cao}^{1}$ \\ Department of Computer \\ Science and Engineering of \\ Tianjin University, China
}

\begin{abstract}
The $n$-gram model is a stochastic model, which predicts the next word (predicted word) given the previous words (conditional words) in a word sequence. The cluster $n$-gram model is a variant of the $n$-gram model in which similar words are classified in the same cluster. It has been demonstrated that using different clusters for predicted and conditional words leads to cluster models that are superior to classical cluster models which use the same clusters for both words. This is the basis of the asymmetric cluster model (ACM) discussed in our study. In this paper, we first present a formal definition of the ACM. We then describe in detail the methodology of constructing the ACM. The effectiveness of the ACM is evaluated on a realistic application, namely Japanese Kana-Kanji conversion. Experimental results show substantial improvements of the ACM in comparison with classical cluster models and word $n$-gram models at the same model size. Our analysis shows that the high-performance of the ACM lies in the asymmetry of the model.
\end{abstract}

\section{Introduction}

The $n$-gram model has been widely applied in many applications such as speech recognition, machine translation, and Asian language text input [Jelinek, 1990; Brown et al., 1990; Gao et al., 2002]. It is a stochastic model, which predicts the next word (predicted word) given the previous $n-1$ words (conditional words) in a word sequence.

\author{
Joshua T. Goodman \\ Microsoft Research, Redmond \\ Washington 98052, USA \\ joshuago@microsoft.com \\ Hang Li \\ Microsoft Research, Asia \\ Beijing, 100080, P.R.C \\ hangli@microsoft. com
}

The cluster $n$-gram model is a variant of the word $n$-gram model in which similar words are classified in the same cluster. This has been demonstrated as an effective way to deal with the data sparseness problem and to reduce the memory sizes for realistic applications. Recent research [Yamamoto et al., 2001] shows that using different clusters for predicted and conditional words can lead to cluster models that are superior to classical cluster models, which use the same clusters for both words [Brown et al., 1992]. This is the basis of the asymmetric cluster model (ACM), which will be formally defined and empirically studied in this paper. Although similar models have been used in previous studies [Goodman and Gao, 2000; Yamamoto et al., 2001], several issues have not been completely investigated. These include: (1) an effective methodology for constructing the ACM, (2) a thorough comparative study of the ACM with classical cluster models and word models when they are applied to a realistic application, and (3) an analysis of the reason why the ACM is superior.

The goal of this study is to address the above three issues. We first present a formal definition of the ACM; then we describe in detail the methodology of constructing the ACM including (1) an asymmetric clustering algorithm in which different metrics are used for clustering the predicted and conditional words respectively; and (2) a method for model parameter optimization in which the optimal cluster numbers are found for different clusters. We evaluate the ACM on a real application, Japanese Kana-Kanji conversion, which converts phonetic Kana strings into proper Japanese orthography. The performance is measured in terms of character error rate (CER). Our results show substantial improvements of the ACM in comparison with classical cluster models and word $n$-gram models at the same model size. Our analysis shows that the high-performance of the ACM comes

\footnotetext{
1 This work was done while Cao was visiting Microsoft Research Asia.
} 
from better structure and better smoothing, both of which lie in the asymmetry of the model.

This paper is organized as follows: Section 1 introduces our research topic, and then Section 2 reviews related work. Section 3 defines the ACM and describes in detail the method of model construction. Section 4 first introduces the Japanese Kana-Kanji conversion task; it then presents our main experiments and a discussion of our findings. Finally, conclusions are presented in Section 5.

\section{Related Work}

A large amount of previous research on clustering has been focused on how to find the best clusters [Brown et al., 1992; Kneser and Ney, 1993; Yamamoto and Sagisaka, 1999; Ueberla, 1996; Pereira et al., 1993; Bellegarda et al., 1996; Bai et al., 1998]. Only small differences have been observed, however, in the performance of the different techniques for constructing clusters. In this study, we focused our research on novel techniques for using clusters - the ACM, in which different clusters are used for predicted and conditional words respectively.

The discussion of the ACM in this paper is an extension of several studies below. The first similar cluster model was presented by Goodman and Gao [2000] in which the clustering techniques were combined with Stolcke's [1998] pruning to reduce the language model (LM) size effectively. Goodman [2001] and Gao et al, [2001] give detailed descriptions of the asymmetric clustering algorithm. However, the impact of the asymmetric clustering on the performance of the resulting cluster model was not empirically studied there. Gao et al., [2001] presented a fairly thorough empirical study of clustering techniques for Asian language modeling. Unfortunately, all of the above work studied the ACM without applying it to an application; thus only perplexity results were presented. The first real application of the ACM was a simplified bigram ACM used in a Chinese text input system [Gao et al. 2002]. However, quite a few techniques (including clustering) were integrated to construct a Chinese language modeling system, and the contribution of using the ACM alone was by no means completely investigated.

Finally, there is one more point worth mentioning. Most language modeling improvements reported previously required significantly more space than word trigram models [Rosenfeld, 2000]. Their practical value is questionable since all realistic applications have memory constraints. In this paper, our goal is to achieve a better tradeoff between LM performance (perplexity and CER) and model size. Thus, whenever we compare the performance of different models (i.e. ACM vs. word trigram model), Stolcke's pruning is employed to bring the models compared to similar sizes.

\section{Asymmetric Cluster Model}

\subsection{Model}

The LM predicts the next word $w_{i}$ given its history $h$ by estimating the conditional probability $P\left(w_{i} \mid h\right)$. Using the trigram approximation, we have $P\left(w_{i} \mid h\right) \approx P\left(w_{i} \mid w_{i-2} w_{i-1}\right)$, assuming that the next word depends only on the two preceding words.

In the ACM, we will use different clusters for words in different positions. For the predicted word, $w_{i}$, we will denote the cluster of the word by $P W_{i}$, and we will refer to this as the predictive cluster. For the words $w_{i-2}$ and $w_{i-1}$ that we are conditioning on, we will denote their clusters by $C W_{i-2}$ and $C W_{i-1}$ which we call conditional clusters. When we which to refer to a cluster of a word $w$ in general we will use the notation $W$. The ACM estimates the probability of $w_{i}$ given the two preceeding words $w_{i-2}$ and $w_{i-1}$ as the product of the following two probabilities:

(1) The probability of the predicted cluster $P W_{i}$ given the preceding conditional clusters $C W_{i-2}$ and $C W_{i-1}, P\left(P W_{i} \mid C W_{i-2} C W_{i-1}\right)$, and

(2) The probability of the word given its cluster $P W_{i}$ and the preceding conditional clusters $C W_{i-2}$ and $C W_{i-1}, P\left(w_{i} \mid C W_{i-2} C W_{i-1} P W_{i}\right)$.

Thus, the ACM can be parameterized by

$P\left(w_{i} \mid h\right) \approx P\left(P W_{i} \mid C W_{i-2} C W_{i-1}\right) \times P\left(w_{i} \mid C W_{i-2} C W_{i-1} P W_{i}\right)$

The ACM consists of two sub-models: (1) the cluster sub-model $P\left(P W_{i} \mid C W_{i-2} C W_{i-1}\right)$, and (2) the word sub-model $P\left(w_{i} \mid C W_{i-2} C W_{i-1} P W_{i}\right)$. To deal with the data sparseness problem, we used a backoff scheme (Katz, 1987) for the parameter estimation of each sub-model. The backoff scheme recursively estimates the probability of an unseen $n$-gram by utilizing $(n-1)$-gram estimates.

The basic idea underlying the ACM is the use of different clusters for predicted and conditional words respectively. Classical cluster models are symmetric in that the same clusters are employed for both predicted and conditional words. However, the symmetric cluster model is suboptimal in practice. For example, consider a pair of words like "a" and "an". In general, "a" and "an" can follow the same words, and thus, as predicted words, belong in the same cluster. But, there are very few words that can 
follow both "a" and "an". So as conditional words, they belong in different clusters.

In generating clusters, two factors need to be considered: (1) clustering metrics, and (2) cluster numbers. In what follows, we will investigate the impact of each of the factors.

\subsection{Asymmetric clustering}

The basic criterion for statistical clustering is to maximize the resulting probability (or minimize the resulting perplexity) of the training data. Many traditional clustering techniques [Brown et al., 1992] attempt to maximize the average mutual information of adjacent clusters

$$
I\left(W_{1}, W_{2}\right)=\sum_{W_{1}, W_{2}} P\left(W_{1} W_{2}\right) \log \frac{P\left(W_{2} \mid W_{1}\right)}{P\left(W_{2}\right)},
$$

where the same clusters are used for both predicted and conditional words. We will call these clustering techniques symmetric clustering, and the resulting clusters both clusters. In constructing the ACM, we used asymmetric clustering, in which different clusters are used for predicted and conditional words. In particular, for clustering conditional words, we try to minimize the perplexity of training data for a bigram of the form $P\left(w_{i} \mid W_{i-1}\right)$, which is equivalent to maximizing

$$
\prod_{i=1}^{N} P\left(w_{i} \mid W_{i-1}\right) .
$$

where $N$ is the total number of words in the training data. We will call the resulting clusters conditional clusters denoted by $C W$. For clustering predicted words, we try to minimize the perplexity of training data of $P\left(W_{i} \mid w_{i-1}\right) \times P\left(w_{i} \mid W_{i}\right)$. We will call the resulting clusters predicted clusters denoted by $P W$. We have ${ }^{2}$

$$
\begin{aligned}
\prod_{i=1}^{N} P\left(W_{i} \mid w_{i-1}\right) \times P\left(w_{i} \mid W_{i}\right) & =\prod_{i=1}^{N} \frac{P\left(w_{i-1} W_{i}\right)}{P\left(w_{i-1}\right)} \times \frac{P\left(W_{i} w_{i}\right)}{P\left(W_{i}\right)} \\
& =\prod_{i=1}^{N} \frac{P\left(W_{i} w_{i}\right)}{P\left(w_{i-1}\right)} \times \frac{P\left(w_{i-1} W_{i}\right)}{P\left(W_{i}\right)} \\
& =\prod_{i=1}^{N} \frac{P\left(w_{i}\right)}{P\left(w_{i-1}\right)} \times P\left(w_{i-1} \mid W_{i}\right) .
\end{aligned}
$$

Now, $\frac{P\left(w_{i}\right)}{P\left(w_{i-1}\right)}$ is independent of the clustering used.

Therefore, for the selection of the best clusters, it is sufficient to try to maximize

$$
\prod_{i=1}^{N} P\left(w_{i-1} \mid W_{i}\right) \cdot
$$

This is very convenient since it is exactly the opposite of what was done for conditional clustering. It

\footnotetext{
${ }^{2}$ Thanks to Lillian Lee for suggesting this justification of
} predictive clusters. means that we can use the same clustering tool for both, and simply switch the order used by the program used to get the raw counts for clustering.

The clustering technique we used creates a binary branching tree with words at the leaves. The ACM in this study is a hard cluster model, meaning that each word belongs to only one cluster. So in the clustering tree, each word occurs in a single leaf. In the ACM, we actually use two different clustering trees. One is optimized for predicted words, and the other for conditional words.

The basic approach to clustering we used is a top-down, splitting clustering algorithm. In each iteration, a cluster is split into two clusters in the way that the splitting achieves the maximal entropy decrease (estimated by Equations (3) or (4)). Finally, we can also perform iterations of swapping all words between all clusters until convergence i.e. no more entropy decrease can be found ${ }^{3}$. We find that our algorithm is much more efficient than agglomerative clustering algorithms - those which merge words bottom up.

\subsection{Parameter optimization}

Asymmetric clustering results in two binary clustering trees. By cutting the trees at a certain level, it is possible to achieve a wide variety of different numbers of clusters. For instance, if the tree is cut after the $8^{\text {th }}$ level, there will be roughly $2^{8}=256$ clusters. Since the tree is not balanced, the actual number of clusters may be somewhat smaller. We use $W^{l}$ to represent the cluster of a word $w$ using a tree cut at level $l$. In particular, if we set $l$ to the value "all", it means that the tree is cut at infinite depth, i.e. each cluster contains a single word. The ACM model of Equation (1) can be rewritten as

$$
P\left(P W_{i}^{l} \mid C W_{i-2}^{j} C W_{i-1}^{j}\right) \times P\left(w_{i} \mid P W_{i-2}{ }^{k} C W_{i-1}{ }^{k} C W_{i}^{l}\right) \text {. }
$$

To optimally apply the ACM to realistic applications with memory constraints, we are always seeking the correct balance between model size and performance. We used Stolcke's pruning method to produce many ACMs with different model sizes. In our experiments, whenever we compare techniques, we do so by comparing the performance (perplexity and CER) of the LM techniques at the same model sizes. Stolcke's pruning is an entropy-based cutoff

\footnotetext{
${ }^{3}$ Notice that for experiments reported in this paper, we used the basic top-down algorithm without swapping. Although the resulting clusters without swapping are not even locally optimal, our experiments show that the quality of clusters (in terms of the perplexity of the resulting $\mathrm{ACM}$ ) is not inferior to that of clusters with swapping.
} 
method, which can be described as follows: all $n$-grams that change perplexity by less than a threshold are removed from the model. For pruning the ACM, we have two thresholds: one for the cluster sub-model $P\left(P W_{i}^{l} \mid C W_{i-2}{ }^{j} C W_{i-1}{ }^{j}\right)$ and one for the word sub-model $P\left(w_{i} \mid C W_{i-2}{ }^{k} C W_{i-1}{ }^{k} P W_{i}^{l}\right)$ respectively, denoted by $t_{c}$ and $t_{w}$ below.

In this way, we have 5 different parameters that need to be simultaneously optimized: $l, j, k, t_{c}$, and $t_{w}$, where $j, k$, and $l$ are the numbers of clusters, and $t_{c}$ and $t_{w}$ are the pruning thresholds.

A brute-force approach to optimizing such a large number of parameters is prohibitively expensive. Rather than trying a large number of combinations of all 5 parameters, we give an alternative technique that is significantly more efficient. Simple math shows that the perplexity of the overall model $P\left(P W_{i}^{l} \mid C W_{i-2}^{j} C W_{i-1}^{j}\right) \times \quad P\left(w_{i} \mid C W_{i-2}{ }^{k} C W_{i-1}{ }^{k} P W_{i}^{l}\right) \quad$ is equal to the perplexity of the cluster sub-model $P\left(P W_{i}^{l} \mid C W_{i-2}^{j} C W_{i-1}^{j}\right)$ times the perplexity of the word sub-model $P\left(w_{i} \mid C W_{i-2}{ }^{k} C W_{i-1}{ }^{k} P W_{i}^{l}\right)$. The size of the overall model is clearly the sum of the sizes of the two sub-models. Thus, we try a large number of values of $j, l$, and a pruning threshold $t_{c}$ for $P\left(P W_{i}^{l} \mid C W_{i-2}^{j} C W_{i-1}^{j}\right)$, computing sizes and perplexities of each, and a similarly large number of values of $l, k$, and a separate threshold $t_{w}$ for $P\left(w_{i} \mid C W_{i-2}{ }^{k} C W_{i-1}{ }^{k} P W_{i}^{l}\right)$. We can then look at all compatible pairs of these models (those with the same value of $l$ ) and quickly compute the perplexity and size of the overall models. This allows us to relatively quickly search through what would otherwise be an overwhelmingly large search space.

\section{Experimental Results and Discussion}

\subsection{Japanese Kana-Kanji Conversion Task}

Japanese Kana-Kanji conversion is the standard method of inputting Japanese text by converting a syllabary-based Kana string into the appropriate combination of ideographic Kanji and Kana. This is a similar problem to speech recognition, except that it does not include acoustic ambiguity. The performance is generally measured in terms of character error rate (CER), which is the number of characters wrongly converted from the phonetic string divided by the number of characters in the correct transcript. The role of the language model is, for all possible word strings that match the typed phonetic symbol string, to select the word string with the highest language model probability. Current products make about $5-10 \%$ errors in conversion of real data in a wide variety of domains.

\subsection{Settings}

In the experiments, we used two Japanese newspaper corpora: the Nikkei Newspaper corpus, and the Yomiuri Newspaper corpus. Both text corpora have been word-segmented using a lexicon containing 167,107 entries.

We performed two sets of experiments: (1) pilot experiments, in which model performance is measured in terms of perplexity and (2) Japanese Kana-Kanji conversion experiments, in which the performance of which is measured in terms of CER.

In the pilot experiments, we used a subset of the Nikkei newspaper corpus: ten million words of the Nikkei corpus for language model training, 10,000 words for held-out data, and 20,000 words for testing data. None of the three data sets overlapped. In the Japanese Kana-Kanji conversion experiments, we built language models on a subset of the Nikkei Newspaper corpus, which contains 36 million words. We performed parameter optimization on a subset of held-out data from the Yomiuri Newspaper corpus, which contains 100,000 words. We performed testing on another subset of the Yomiuri Newspaper corpus, which contains 100,000 words. In both sets of experiments, word clusters were derived from bigram counts generated from the training corpora. Out-of-vocabulary words were not included in perplexity and error rate computations.

\subsection{Impact of asymmetric clustering}

As described in Section 3.2, depending on the clustering metrics we chose for generating clusters, we obtained three types of clusters: both clusters (the metric of Equation (2)), conditional clusters (the metric of Equation (3)), and predicted clusters (the metric of Equation (4)). We then performed a series of experiments to investigate the impact of different types of clusters on the ACM. We used three variants of the trigram ACM: (1) the predictive cluster model $P\left(w_{i} \mid w_{i-2} w_{i-1} W_{i}\right) \times P\left(W_{i} \mid w_{i-2} w_{i-1}\right)$ where only predicted words are clustered, (2) the conditional cluster model $P\left(w_{i} \mid W_{i-2} W_{i-1}\right)$ where only conditional words are clustered, and (3) the IBM model $P\left(w_{i} \mid W_{i}\right) \times P\left(W_{i} \mid W_{i-2} W_{i-1}\right)$ which can be treated as a special case of the ACM of Equation (5) by using the same type of cluster for both predicted and conditional words, and setting $k=0$, and $l=j$. For each cluster trigram model, we compared their perplexities and CER results on Japanese KanaKanji conversion using different types of clusters. For each cluster type, the number of clusters were fixed to the same value $2^{\wedge} 6$ just for comparison. The results are shown in Table 1. It turns out that the benefit of using different clusters in different 
positions is obvious. For each cluster trigram model, the best results were achieved by using the "matched" clusters, e.g. the predictive cluster model $P\left(w_{i} \mid w_{i-2} w_{i-1} W_{i}\right) \times \quad P\left(W_{i} \mid w_{i-2} w_{i-1}\right)$ has the best performance when the cluster $W_{i}$ is the predictive cluster $P W_{i}$ generated by using the metric of Equation (4). In particular, the IBM model achieved the best results when predicted and conditional clusters were used for predicted and conditional words respectively. That is, the IBM model is of the form $P\left(w_{i} \mid P W_{i}\right) \times P\left(P W_{i} \mid C W_{i-2} C W_{i-1}\right)$.

\begin{tabular}{|c|c|c|c|c|c|}
\hline \multicolumn{2}{|c|}{} & Con & Pre & Both & Con + Pre \\
\hline \multirow{2}{*}{$\begin{array}{c}\text { Con } \\
\text { model }\end{array}$} & Perplexity & $\mathbf{2 8 7 . 7}$ & 414.5 & 377.6 & --- \\
\cline { 2 - 6 } & CER (\%) & $\mathbf{4 . 5 8}$ & 11.78 & 12.56 & --- \\
\hline $\begin{array}{c}\text { Pre } \\
\text { model }\end{array}$ & Perplexity & 103.4 & $\mathbf{1 0 2 . 4}$ & 103.3 & --- \\
\cline { 2 - 6 } & CER (\%) & 3.92 & $\mathbf{3 . 6 3}$ & 3.82 & --- \\
\hline $\begin{array}{c}\text { IBM } \\
\text { model }\end{array}$ & Perplexity & 548.2 & 514.4 & $\mathbf{3 8 5 . 2}$ & $\mathbf{3 8 2 . 2}$ \\
\cline { 2 - 6 } & CER (\%) & 6.61 & 6.49 & $\mathbf{5 . 8 2}$ & $\mathbf{5 . 3 6}$ \\
\hline
\end{tabular}

Table 1: Comparison of different cluster types with cluster-based models

\subsection{Impact of parameter optimization}

In this section, we first present our pilot experiments of finding the optimal parameter set of the $\operatorname{ACM}(l, j$, $\left.k, t_{c}, t_{w}\right)$ described in Section 2.3. Then, we compare the ACM to the IBM model, showing that the superiority of the ACM results from its better structure.

In this section, the performance of LMs was measured in terms of perplexity, and the size was measured as the total number of parameters of the LM: one parameter for each bigram and trigram, one parameter for each normalization parameter $\alpha$ that was needed, and one parameter for each unigram.

We first used the conditional cluster model of the form $P\left(w_{i} \mid C W_{i-2}{ }^{j} C W_{i-1}{ }^{j}\right)$. Some sample settings of parameters $\left(j, t_{w}\right)$ are shown in Figure 1. The performance was consistently improved by increasing the number of clusters $j$, except at the smallest sizes. The word trigram model was consistently the best model, except at the smallest sizes, and even then was only marginally worse than the conditional cluster models. This is not surprising because the conditional cluster model always discards information for predicting words.

We then used the predictive cluster model of the form $P\left(P W_{i}^{l} \mid w_{i-2} w_{i-1}\right) \times P\left(w_{i} \mid w_{i-2} w_{i-1} P W_{i}^{l}\right)$, where only predicted words are clustered. Some sample settings of the parameters $\left(l, t_{c}, t_{w}\right)$ are shown in Figure 2. For simplicity, we assumed $t_{c}=t_{w}$, meaning that the same pruning threshold values were used for both sub-models. It turns out that predictive cluster models achieve the best perplexity results at about $2^{\wedge} 6$ or $2^{\wedge} 8$ clusters. The models consistently outperform the baseline word trigram models.

We finally returned to the ACM of Equation (5), where both conditional words and the predicted word are clustered (with different numbers of clusters), and which is referred to as the combined cluster model below. In addition, we allow different values of the threshold for different sub-models. Therefore, we need to optimize the model parameter set $l, j, k, t_{c}, t_{w}$.

Based on the pilot experiment results using conditional and predictive cluster models, we tried combined cluster models for values $l \in[4,10], j$, $k \in[8,16]$. We also allow $j, k=$ all. Rather than plot all points of all models together, we show only the outer envelope of the points. That is, if for a given model type and a given point there is some other point of the same type with both lower perplexity and smaller size than the first point, then we do not plot the first, worse point.

The results are shown in Figure 3, where the cluster number of IBM models is $2^{\wedge} 14$ which achieves the best performance for IBM models in our experiments. It turns out that when $l \in[6,8]$ and $j, k>12$, combined cluster models yield the best results. We also found that the predictive cluster models give as good performance as the best combined ones while combined models outperformed very slightly only when model sizes are small. This is not difficult to explain. Recall that the predictive cluster model is a special case of the combined model where words are used in conditional positions, i.e. $j=k=$ all. Our experiments show that combined models achieved good performance when large numbers of clusters are used for conditional words, i.e. large $j, k>12$, which are similar to words.

The most interesting analysis is to look at some sample settings of the parameters of the combined cluster models in Figure 3. In Table 2, we show the best parameter settings at several levels of model size. Notice that in larger model sizes, predictive cluster models (i.e. $j=k=$ all) perform the best in some cases. The 'prune' columns (i.e. columns 6 and 7) indicate the Stolcke pruning parameter we used.

First, notice that the two pruning parameters (in columns 6 and 7) tend to be very similar. This is desirable since applying the theory of relative entropy pruning predicts that the two pruning parameters should actually have the same value.

Next, let us compare the ACM $P\left(P W_{i}^{l} \mid C W_{i-2}^{j} C W_{i-1}^{j}\right) \times P\left(w_{i} \mid C W_{i-2}^{k} C W_{i-1}{ }^{k} P W_{i}^{l}\right)$ to traditional IBM clustering of the form $P\left(W_{i}^{l} \mid W_{i-2}^{l} W_{i-1}^{l}\right) \times P\left(w_{i} \mid W_{i}^{l}\right)$, which is equal to $P\left(W_{i}^{l} \mid W_{i-2}{ }^{l} W_{i-1}{ }^{l}\right) \times P\left(w_{i} \mid W_{i-2}{ }^{0} W_{i-1}{ }^{0} W_{i}^{l}\right)$ (assuming the 


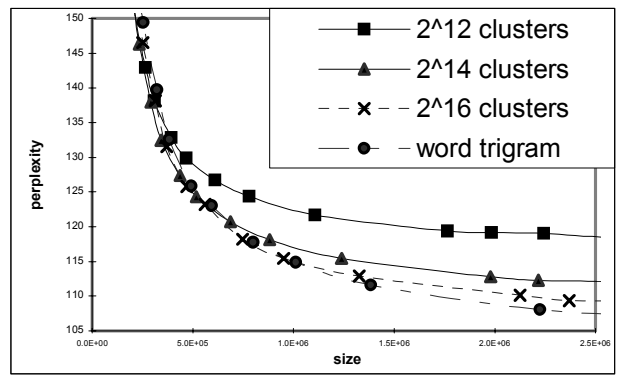

Figure 1. Comparison of conditional models applied with different numbers of clusters

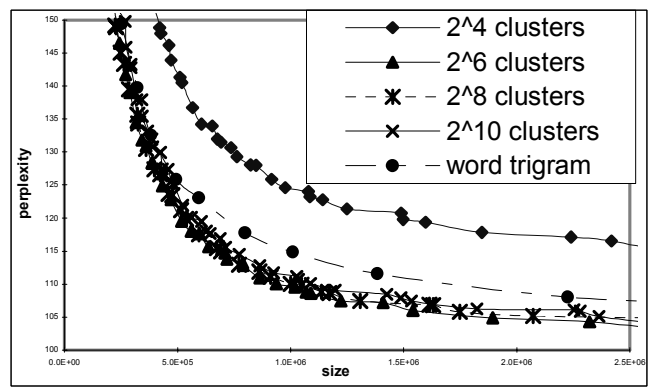

Figure 2. Comparison of predictive models applied with different numbers of clusters

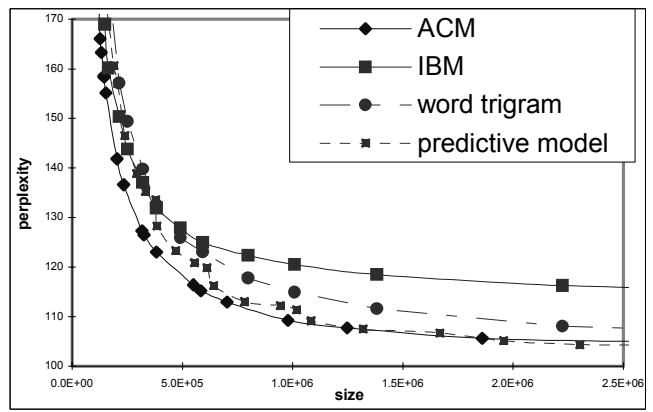

Figure 3. Comparison of ACMs, predictive cluster model, IBM model, and word trigram model

same type of cluster is used for both predictive and conditional words). Our results in Figure 3 show that the performance of IBM models is roughly an order of magnitude worse than that of ACMs. This is because in addition to the use of the symmetric cluster model, the traditional IBM model makes two more assumptions that we consider suboptimal. First, it assumes that $j=l$. We see that the best results come from unequal settings of $j$ and $l$. Second, more importantly, IBM clustering assumes that $k=0$. We see that not only is the optimal setting for $k$ not 0 , but also typically the exact opposite is the optimal: $k=$ all in which case $P\left(w_{i} \mid C W_{i-2}{ }^{k} C W_{i-1}{ }^{k} P W_{i}^{l}\right)=$ $P\left(w_{i} \mid w_{i-2} w_{i-1} P W_{i}^{l}\right)$, or $k=14,16$, which is very similar. That is, we see that words depend on the previous words and that an independence assumption is a poor one. Of course, many of these word dependencies are pruned away - but when a word does depend on something, the previous words are better predictors than the previous clusters.

Another important finding here is that for most of these settings, the unpruned model is actually larger than a normal trigram model - whenever $k=$ all or 14 , 16, the unpruned model $P\left(P W_{i}^{l} \mid C W_{i-2}{ }^{j} C W_{i-1}{ }^{j}\right) \times$ $P\left(w_{i} \mid C W_{i-2}{ }^{k} C W_{i-1}{ }^{k} P W_{i}^{l}\right)$ is actually larger than an unpruned model $P\left(w_{i} \mid w_{i-2} w_{i-1}\right)$.

This analysis of the data is very interesting - it implies that the gains from clustering are not from compression, but rather from capturing structure. Factoring the model into two models, in which the cluster is predicted first, and then the word is predicted given the cluster, allows the structure and regularities of the model to be found. This larger, better structured model can be pruned more effectively, and it achieved better performance than a word trigram model at the same model size.

\begin{tabular}{|c|c|c|c|c|c|c|}
\hline Model size & Perplexity & $l$ & $j$ & $k$ & $t_{c}$ & $t_{w}$ \\
\hline $2.0 \mathrm{E}+05$ & 141.1 & 8 & 12 & 14 & 24 & 24 \\
\hline $2.5 \mathrm{E}+05$ & 135.7 & 8 & 12 & 14 & 12 & 24 \\
\hline $5.0 \mathrm{E}+05$ & 118.8 & 6 & 14 & 16 & 6 & 12 \\
\hline $7.5 \mathrm{E}+05$ & 112.8 & 6 & 16 & 16 & 3 & 6 \\
\hline $1.0 \mathrm{E}+06$ & 109.0 & 6 & 16 & 16 & 3 & 3 \\
\hline $1.3 \mathrm{E}+06$ & 107.4 & 6 & 16 & 16 & 2 & 3 \\
\hline $1.5 \mathrm{E}+06$ & 106.0 & 6 & All & all & 2 & 2 \\
\hline $1.9 \mathrm{E}+06$ & 104.9 & 6 & All & all & 1 & 2 \\
\hline
\end{tabular}

Table 2: Sample parameter settings for the ACM

\subsection{CER results}

Before we present CER results of the Japanese Kana-Kanji conversion system, we briefly describe our method for storing the ACM in practice.

One of the most common methods for storing backoff $n$-gram models is to store $n$-gram probabilities (and backoff weights) in a tree structure, which begins with a hypothetical root node that branches out into unigram nodes at the first level of the tree, and each of those unigram nodes in turn branches out into bigram nodes at the second level and so on. To save storage, $n$-gram probabilities such as $P\left(w_{i} \mid w_{i-1}\right)$ and backoff weights such as $\alpha\left(w_{i-2} w_{i-1}\right)$ are stored in a single (bigram) node array (Clarkson and Rosenfeld, 1997).

Applying the above tree structure to storing the $\mathrm{ACM}$ is a bit complicated - there are some representation issues. For example, consider the cluster sub-model $P\left(P W_{i}^{l} \mid C W_{i-2}{ }^{j} C W_{i-1}{ }^{j}\right)$. $\quad \mathrm{N}$-gram probabilities such as $P\left(P W_{i}^{l} \mid C W_{i-1}^{j}\right)$ and backoff weights such as $\alpha\left(C W_{i-2}{ }^{j} C W_{i-1}{ }^{j}\right)$ cannot be stored in a single (bigram) node array, because $l \neq j$ and 
$P W \neq C W$. Therefore, we used two separate trees to store probabilities and backoff weights, respectively. As a result, we used four tree structures to store ACMs in practice: two for the cluster sub-model $P\left(P W_{i}^{l} \mid C W_{i-2}{ }^{j} C W_{i-1}{ }^{j}\right)$, and two for the word sub-model $P\left(w_{i} \mid C W_{i-2}{ }^{k} C W_{i-1}{ }^{k} P W_{i}^{l}\right)$. We found that the effect of the storage structure cannot be ignored in a real application.

In addition, we used several techniques to compress model parameters (i.e. word id, $n$-gram probability, and backoff weight, etc.) and reduce the storage space of models significantly. For example, rather than store 4-byte floating point values for all $n$-gram probabilities and backoff weights, the values are quantized to a small number of quantization levels. Quantization is performed separately on each of the $n$-gram probability and backoff weight lists, and separate quantization level look-up tables are generated for each of these sets of parameters. We used 8-bit quantization, which shows no performance decline in our experiments.

Our goal is to achieve the best tradeoff between performance and model size. Therefore, we would like to compare the ACM with the word trigram model at the same model size. Unfortunately, the ACM contains four sub-models and this makes it difficult to be pruned to a specific size. Thus for comparison, we always choose the ACM with smaller size than its competing word trigram model to guarantee that our evaluation is under-estimated. Experiments show that the ACMs achieve statistically significant improvements over word trigram models at even smaller model sizes ( $\mathrm{p}$-value $=8.0 \mathrm{E}-9)$. Some results are shown in Table 3 .

\begin{tabular}{|c|c|c|c|c|}
\hline \multicolumn{2}{|c|}{ Word trigram model } & \multicolumn{3}{|c|}{ ACM } \\
\hline $\begin{array}{c}\text { Size } \\
\text { (MB) }\end{array}$ & CER & $\begin{array}{c}\text { Size } \\
\text { (MB) }\end{array}$ & CER & $\begin{array}{c}\text { CER } \\
\text { Reduction }\end{array}$ \\
\hline 1.8 & $4.56 \%$ & 1.7 & $4.25 \%$ & $6.8 \%$ \\
\hline 5.8 & $4.08 \%$ & 4.5 & $3.83 \%$ & $6.1 \%$ \\
\hline 11.7 & $4.04 \%$ & 10.7 & $3.73 \%$ & $7.7 \%$ \\
\hline 23.5 & $4.00 \%$ & 21.7 & $3.63 \%$ & $9.3 \%$ \\
\hline 42.4 & $3.98 \%$ & 40.4 & $3.63 \%$ & $8.8 \%$ \\
\hline
\end{tabular}

Table 3: CER results of ACMs and word trigram models at different model sizes

Now we discuss why the ACM is superior to simple word trigrams. In addition to the better structure as shown in Section 3.3, we assume here that the benefit of our model also comes from its better smoothing. Consider a probability such as $P($ Tuesday $\mid$ party on $)$. If we put the word "Tuesday" into the cluster WEEKDAY, we decompose the probability
$P($ Tuesday $\mid$ party on $)=P($ WEEKDAY $\mid$ party on $) \times$ $P($ Tuesday | party on WEEKDAY $)$.

When each word belongs to one class, simple math shows that this decomposition is a strict equality. However, when smoothing is taken into consideration, using the clustered probability will be more accurate than using the non-clustered probability. For instance, even if we have never seen an example of "party on Tuesday", perhaps we have seen examples of other phrases, such as "party on Wednesday"; thus, the probability $P(W E E K D A Y$ party on) will be relatively high. Furthermore, although we may never have seen an example of "party on WEEKDAY Tuesday", after we backoff or interpolate with a lower order model, we may able to accurately estimate $P($ Tuesday | on WEEKDAY). Thus, our smoothed clustered estimate may be a good one.

Our assumption can be tested empirically by following experiments. We first constructed several test sets with different backoff rates ${ }^{4}$. The backoff rate of a test set, when presented to a trigram model, is defined as the number of words whose trigram probabilities are estimated by backoff bigram probabilities divided by the number of words in the test set. Then for each test set, we obtained a pair of CER results using the ACM and the word trigram model respectively. As shown in Figure 4, in both cases, CER increases as the backoff rate increases from $28 \%$ to $40 \%$. But the curve of the word trigram model has a steeper upward trend. The difference of the upward trends of the two curves can be shown more clearly by plotting the CER difference between them, as shown in Figure 5. The results indicate that because of its better smoothing, when the backoff rate increases, the CER using the ACM does not increase as fast as that using the word trigram model. Therefore, we are reasonably confident that some portion of the benefit of the ACM comes from its better smoothing.

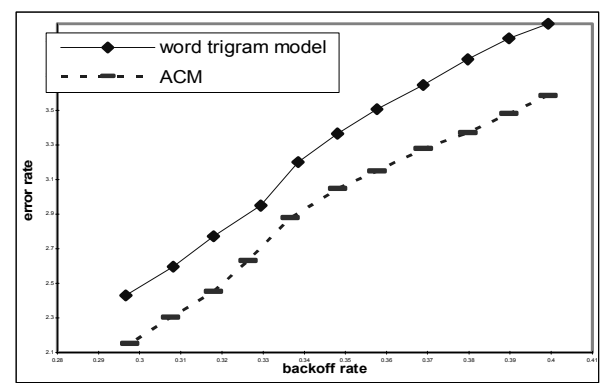

Figure 4: CER vs. backoff rate.

\footnotetext{
4 The backoff rates are estimated using the baseline trigram model, so the choice could be biased against the word trigram model.
} 


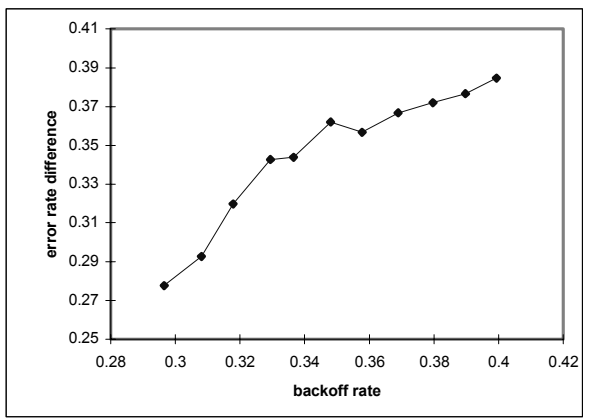

Figure 5: CER difference vs. backoff rate.

\section{Conclusion}

There are three main contributions of this paper. First, after presenting a formal definition of the ACM, we described in detail the methodology of constructing the ACM effectively. We showed empirically that both the asymmetric clustering and the parameter optimization (i.e. optimal cluster numbers) have positive impacts on the performance of the resulting ACM. The finding demonstrates partially the effectiveness of our research focus: techniques for using clusters (i.e. the ACM) rather than techniques for finding clusters (i.e. clustering algorithms). Second, we explored the actual representation of the ACM and evaluate it on a realistic application - Japanese Kana-Kanji conversion. Results show approximately 6-10\% $\mathrm{CER}$ reduction of the ACMs in comparison with the word trigram models, even when the ACMs are slightly smaller. Third, the reasons underlying the superiority of the ACM are analyzed. For instance, our analysis suggests the benefit of the ACM comes partially from its better structure and its better smoothing.

All cluster models discussed in this paper are based on hard clustering, meaning that each word belongs to only one cluster. One area we have not explored is the use of soft clustering, where a word $w$ can be assigned to multiple clusters $W$ with a probability $P(W \mid w)$ [Pereira et al., 1993]. Saul and Pereira [1997] demonstrated the utility of soft clustering and concluded that any method that assigns each word to a single cluster would lose information. It is an interesting question whether our techniques for hard clustering can be extended to soft clustering. On the other hand, soft clustering models tend to be larger than hard clustering models because a given word can belong to multiple clusters, and thus a training instance $P\left(w_{i} \mid w_{i-2} w_{i-1}\right)$ can lead to multiple counts instead of just 1 .

\section{References}

Bai, S., Li, H., Lin, Z., and Yuan, B. (1998). Building class-based language models with contextual statistics. In ICASSP-98, pp. 173-176.

Bellegarda, J. R., Butzberger, J. W., Chow, Y. L., Coccaro, N. B., and Naik, D. (1996). A novel word clustering algorithm based on latent semantic analysis. In ICASSP-96.

Brown, P. F., Cocke, J., DellaPietra, S. A., DellaPietra, V. J., Jelinek, F., Lafferty, J. D., Mercer, R. L., and Roossin, P. S. (1990). A statistical approach to machine translation. Computational Linguistics, 16(2), pp. 79-85.

Brown, P. F., DellaPietra V. J., deSouza, P. V., Lai, J. C., and Mercer, R. L. (1992). Class-based n-gram models of natural language. Computational Linguistics, 18(4), pp. 467-479.

Clarkson, P. R., and Rosenfeld, R. (1997). Statistical language modeling using the CMU-Cambridge toolkit. In Eurospeech 1997, Rhodes, Greece.

Gao, J. Goodman, J. and Miao, J. (2001). The use of clustering techniques for language model - application to Asian language. Computational Linguistics and Chinese Language Processing. Vol. 6, No. 1, pp 27-60.

Gao, J., Goodman, J., Li, M., and Lee, K. F. (2002). Toward a unified approach to statistical language modeling for Chinese. ACM Transactions on Asian Language Information Processing. Vol. 1, No. 1, pp 3-33.

Goodman, J. (2001). A bit of progress in language modeling. In Computer Speech and Language, October 2001, pp 403-434.

Goodman, J., and Gao, J. (2000). Language model size reduction by predictive clustering. ICSLP-2000, Beijing.

Jelinek, F. (1990). Self-organized language modeling for speech recognition. In Readings in Speech Recognition, A. Waibel and K. F. Lee, eds., Morgan-Kaufmann, San Mateo, CA, pp. 450-506.

Katz, S. M. (1987). Estimation of probabilities from sparse data for the language model component of a speech recognizer. IEEE Transactions on Acoustics, Speech and Signal Processing, ASSP-35(3):400-401, March.

Kneser, R. and Ney, H. (1993). Improved clustering techniques for class-based statistical language modeling. In Eurospeech, Vol. 2, pp. 973-976, Berlin, Germany.

Ney, H., Essen, U., and Kneser, R. (1994). On structuring probabilistic dependences in stochastic language modeling. Computer, Speech, and Language, 8:1-38.

Pereira, F., Tishby, N., and Lee L. (1993). Distributional clustering of English words. In Proceedings of the $31^{\text {st }}$ Annual Meeting of the ACL.

Rosenfeld, R. (2000). Two decades of statistical language modeling: where do we go from here. In Proceeding of the IEEE, 88:1270-1278, August.

Saul, L., and Pereira, F.C.N. (1997). Aggregate and mixed-order Markov models for statistical language processing. In EMNLP-1997.

Stolcke, A. (1998). Entropy-based Pruning of Backoff Language Models. Proc. DARPA News Transcription and Understanding Workshop, 1998, pp. 270-274.

Ueberla, J. P. (1996). An extended clustering algorithm for statistical language models. IEEE Transactions on Speech and Audio Processing, 4(4): 313-316.

Yamamoto, H., Isogai, S., and Sagisaka, Y. (2001). Multi-Class Composite N-gram Language Model for Spoken Language Processing Using Multiple Word Clusters. $39^{\text {th }}$ Annual meetings of the Association for Computational Linguistics (ACL'01), Toulouse, 6-11 July 2001.

Yamamoto, H., and Sagisaka, Y. (1999). Multi-class Composite $\mathrm{N}$-gram based on Connection Direction, In Proceedings of the IEEE International Conference on Acoustics, Speech and Signal Processing, May, Phoenix, Arizona. 